\title{
Combined Online and Delayed Coordinated Charging of Plug-In Electric Vehicles Considering Wind and Rooftop PV Generations
}

\author{
Amir S. Masoum ${ }^{1}$ A. Abu-Siada ${ }^{1} \cdot$ Syed Islam $^{1}$
}

Received: 8 July 2016 / Accepted: 26 September 2016 / Published online: 11 October 2016

(c) Springer Science+Business Media Singapore 2016

\begin{abstract}
Emerging smart grid technology is creating unique opportunity for wide applications of renewable distributed generation resources, smart appliances and plugin electric vehicles (PEVs). This paper presents a combined online (real-time) and delayed (overnight and next day) PEV coordination scheme based on fuzzy reasoning and maximum sensitivity selections (MSS) considering wind and rooftop PV generations. The proposed combined online fuzzy-based and delayed MSS-based (OL-F/DLMSS) algorithm aims at reducing energy generation cost by charging the electric vehicles during high renewable energy generation periods. To include vehicle owners' satisfaction, they are classified into three groups; high priority consumers requiring expensive PEV charging as soon as arriving home during early evening hours, low priority consumers requesting cheap overnight PEV charging and medium priority consumers requiring inexpensive PEV charging within 24 hours. PEV coordination is performed to minimize the cost of generating energy and regulate node voltages while utilizing the available renewable energy resources for vehicle battery charging. Detailed simulations for uncoordinated and combined online/overnight coordinated PEV charging are presented for a 449 node smart grid
\end{abstract}

Amir S. Masoum

a_masoum@ieee.org

A. Abu-Siada

a.abusiada@curtin.edu.au

Syed Islam

s.islam@curtin.edu.au

1 Centre for Smart Grid and Sustainable Power Systems, Department of Electrical and Computer Engineering, Curtin University, Perth, WA, Australia test system consisting of the IEEE 31 node medium voltage distribution network, 3 wind turbines and 22 low voltage 19-node residential feeders with 88 rooftop PVs and 264 PEVs.

Keywords Smart grid · Plug-in electric vehicles . Coordinated charging $\cdot$ Distributed generation

\section{Introduction}

The global trend to develop smart grids (SGs) with bidirectional communications, smart metering, real time monitoring and control is providing new opportunities for the enhancement of the distribution network performance by utilizing more renewables distribution generations (DGs) and new cohort of time variant loads such as plug-in electric vehicles (PEVs) and smart appliances. However, there are still unsolved issues with the transformation from conventional centralized power networks to decentralized producer/user controlled SG configuration particularly with high penetration level of renewable energy sources and PEVs. Among various techniques to improve SG performance is customer demand side management (DSM) using smart metering technology that allows smart appliances, renewable DGs and PEVs to interact with the SG through home area networks for optimum operation and automatic scheduling of their activities at strategic times based on availability and real-time pricing of electrical energy.

References [1-4] present reviews on SG, detrimental impacts of random PEV charging and coordination strategies. Recent studies show that random PEV charging (particularly at high penetration levels) will have detrimental impacts on grid performance and efficiency [2,3]. To solve this issue, electric utilities can either motivate consumers 
to shift their PEV loads to off-peak hours by offering price incentives scheme or by adopting a reliable coordinated PEV charging scheme.

Generally, PEVs can be connected to grid using centralized or decentralized coordination approaches with the energy being transferred from grid to vehicle (G2V) [5-15] or from vehicle to grid (V2G) [2, 16-19]. Reference [9] presents an online PEV coordination algorithm based on maximum sensitivity selections to minimize cost of generating energy for vehicle charging, reduce grid losses and regulate network node voltages. Reference [14] proposes an online fuzzy-based algorithm for PEV coordinated charging to reduce the total cost associated with energy generation and grid losses while maintaining maximum demand levels and node voltage profiles within their permissible limits. Reference [15] proposes an offline overnight MSS based coordinated charging algorithm for PEVs that relies on inexpensive off-peak load hours charging to reduce the cost of generating energy while SG constraints are not violated and all vehicles are fully charged overnight. More recent research on electric vehicle charging include coordination with renewable wind generation [20,21], cost-effective siting of charging infrastructure [22], charging and parking stations [23-25], scenario-based planning of distribution systems with PEVs [26], hierarchical coordinated charging [27] and integration of PEVs in DC distribution system [28], PEV scheduling using load control [29] and marginal-based algorithms [30], as well as modelling [31].

This paper presents a combined online fuzzy-based and delayed MSS-based (OL-F/DL-MSS) algorithm that considers distributed wind and PV generations. The algorithm reduces the cost of energy generation, performs voltage regulation and prevents overloading and revered powering by encouraging and performing PEV charging during offpeak load hours and maximum renewable energy generation periods. Consumers are divided into three groups of high priority (requesting expensive charging during early evening peak load hours), low priority (requesting cheap overnight charging) and medium priority (acquiring inexpensive next day charging). Simulation results are presented and compared with uncoordinated and online/overnight coordinated (OL-FC/ON-MSSC) charging of PEVs populating on a 449 node SG consisting of 3 winds DGs and 22 low voltage, 19node residential feeders with 88 rooftop PVs and 264 PEVs. The locations of rooftop PVs as well as the locations and times of PEVs are randomly assigned but are the same for all simulated cases.

\section{PEV Coordination Problem Formulation}

This section presents the OL-FC algorithm of [14] and the ON-MSSC algorithm of [15] with some modifications and

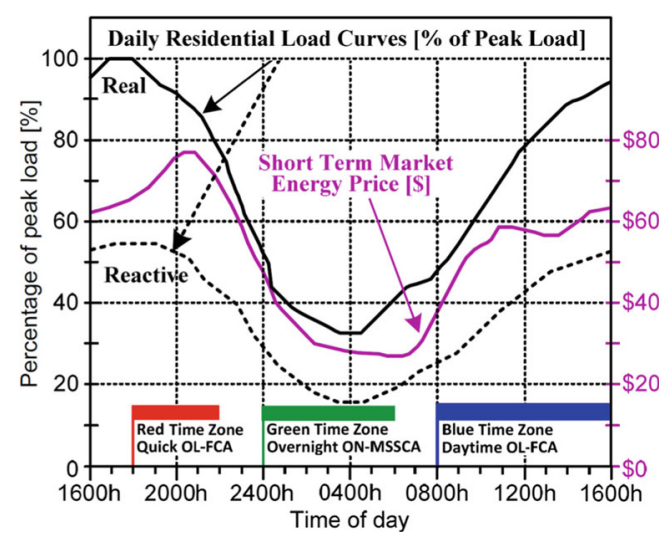

Fig. 1 Proposed subscription priority options of charging time zones, variable short term market energy pricing, typical active and reactive daily residential load curves without PEV charging [9]

improvements to consider renewable generations in PEV coordination.

\section{Online Fuzzy Coordinated (OL-FC) Algorithm for PEV Charging [14]}

The PEV coordination problem is formulated as a nonlinear cost minimization problem with the following objective function and constraints $[9,14]$ :

$$
\begin{aligned}
& \min F_{\cos t}=F_{\cos t-l o s s}+F_{\cos t-g e n}=\sum_{t} K_{E} P_{t, \text { loss }} \\
& +\sum_{t} K_{t, G} D_{t, \text { total }}, \quad t=\Delta t, 2 \Delta t, \ldots .24 \text { hours } \\
& \text { with } P_{t, l o s s}=\sum_{k=0}^{n-1} R_{k, k+1}\left(\left|V_{k+1}-V_{k}\right|\left|y_{k, k+1}\right|\right)^{2} \text {. }
\end{aligned}
$$

Subject to the following constraints:

$\Delta V_{k}=V_{k}-V_{\text {rated }} \leq 0.1 p u$, for $k=1, \ldots, n$

$D_{t, \text { total }}=\sum_{k} P_{t, k}^{\text {load }} \leq D_{t, \max }$

where $F_{\text {cost-gen }}$ and $F_{\text {cost-loss }}$ are the costs corresponding to total generation and total system losses, respectively; $\Delta t=5 \mathrm{~min}$ is the time interval; $K_{E}=50 \$ / \mathrm{MWh}[9,14]$ and $K_{\Delta t, G}$ (Fig. 1) are the costs per MWh of losses and generation, respectively; $k$ and $n$ are respectively the node number and total number of nodes; $\Delta V_{k}$ is the per unit (pu) voltage deviation of bus $k$ which is limited to $0.1 \mathrm{pu}$; while $P_{t, l o s s}, R_{k, k+1}$ and $y_{k, k+1}$ are respectively power loss, resistance and admittance of line section between nodes $k$ and $k+1$ and $D_{t, \max }$ is the maximum demand level (without PEVs) at $t=\Delta t$.

The PEV charging coordination problem of Eqs. 1 and 2 is solved using the OL-FC algorithm of [14] which is based on the MSS optimization of [9] with Fuzzification of constraints given in Eq. 2. MSS optimization approach 
Fig. 2 Fuzzy membership functions for; a voltage deviations (Eqs. 2 and 4), b total system losses (Eqs. 1 and 5), c maximum demand level (Eq. 6), d maximum demand weighting factor (Eq. 6) [14] (a)

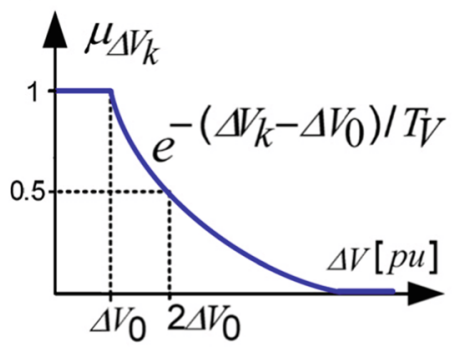

(c)

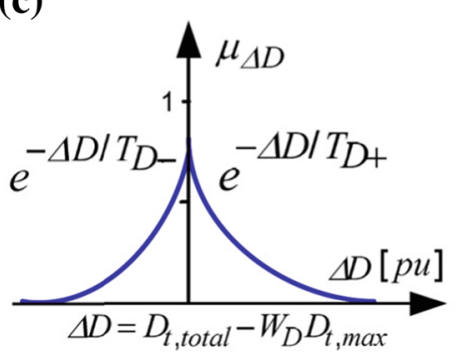

(b)

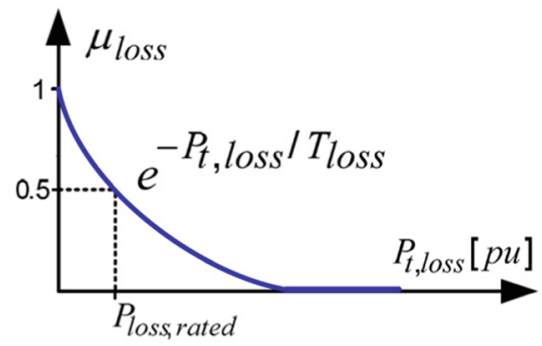

(d)

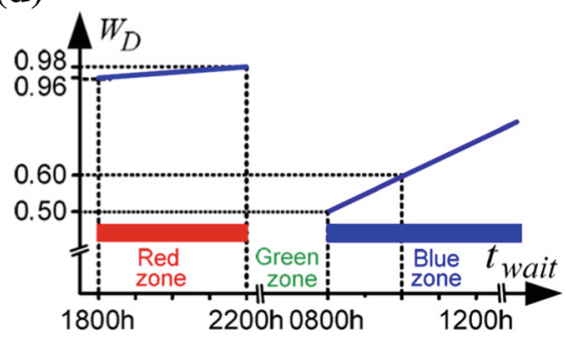

quantifies the sensitivity of Eq. 1 to PEV charging loads at a given time step $t$ :

$M S S_{t, j}=\partial P_{t, l o s s} / \partial P_{P E V, j}, \quad j=1, \ldots ., j_{m}$

where $M S S_{t, j}$ is the sensitivity of system losses to PEV charging at node $j$ at time interval $t$ and $j_{m}$ is the total number of PEVs while $P_{P E V, j}$ is the power consumption of the PEV connected to node $j$.

Equation 3 is also used to sort the plug-in vehicles (at each time interval $t$ ) in the PEV Queue Table. PEVs with higher sensitivities are placed above the table.

The fuzzy reasoning approach of [14] with the fuzzy membership functions of Fig. 2 are used to incorporate the PEV coordination constraints and to select the most suitable PEVs for charging at each time interval $t$.

For the sensitivities of voltage deviations $\Delta V_{k}$ with respect to PEV charging at bus $k$, the exponential membership function $\mu_{\Delta V k}$ of Fig. $2 \mathrm{a}$ is used where $\Delta V_{0}=$ $\Delta V_{\max } / 2$ is selected such that a bus with a voltage deviation less than this limit will have full set memberships while a bus with high voltage deviation will have a low membership value:

$\mu_{\Delta V_{k}}=\left\{\begin{array}{ll}1 & \text { if } \Delta V_{k} \leq \Delta V_{0}, \\ e^{-\left(\Delta V_{k}-\Delta V_{0}\right) / T_{V}} & \text { if } \Delta V_{k}>\Delta V_{0},\end{array} \quad k=1, \ldots, n\right.$

where $\Delta V_{0}=0.05 \mathrm{pu}$ and the time constant is set to $T_{V}=0.034$ such that $\mu_{\Delta V k}=0.23$ for $\Delta V_{k}=$ $\Delta V_{\max }=0.1 \mathrm{pu}$ (Eq. 2).

To limit total system power losses $P_{t, l o s s}$ (Eq. 1) due to PEV charging, the exponential membership function of Fig. $2 b$ is used. The time constant is adjusted such that PEVs charging at time interval $t$ and resulting in system losses less than the rated losses $P_{\text {loss, rated }}$ will have high membership values:

$\mu_{\text {loss }}=e^{-P_{t, l o s s} / T_{\text {loss }}}$

where the time constant is set to $T_{\text {loss }}=0.034$ such that $\mu_{\text {loss }}=0.5$ for total losses equal to the rated losses without any PEV charging $P_{t, \text { loss }}=P_{\text {loss, rated }}$ (Eq. 1).

Two fuzzy exponential membership functions with different time constants are used to limit the maximum total demand $D_{t, l o s s}$ during PEV charging periods as shown in Fig. 2c:

$\mu_{D}= \begin{cases}e^{-\Delta D / T_{D+}} & \text { if } \Delta D=D_{t, \text { total }}-W_{D} D_{t, \max } \geq 0 \\ e^{+\Delta D / T_{D-}} & \text { if } \Delta D=D_{t, \text { total }}-W_{D} D_{t, \max }<0\end{cases}$

where $T_{D+}=0.0125, T_{D-}=0.125[14]$ and $W_{D}$ is the maximum demand weighting factor that will be adjusted based on the vehicle waiting time in the PEV Queue Table (Fig. 2d).

Finally, the algebraic sum of the weighted membership functions is used to combine the fuzzy constraints:

$\mu_{P E V, j}=0.3 \mu_{\Delta V_{k}}+0.3 \mu_{\text {loss }}+0.4 \mu_{D}, \quad j=1, \ldots ., j_{m}$

where constants $0.3,0.3$ and 0.4 are the selected weighting factors for voltage deviation, system loss and maximum demand membership functions, respectively. At each time interval $t$, the decision on whether to start or defer the charging of a vehicle depends on its membership function as well as its ranking in the PEV Queue Table (Eq. 3).

Figure 3 demonstrates how the coordinated charging schedules of PEVs are determined based on Eqs. 1-7. The OL-FC algorithm begins by reading input parameters (such as the load active and reactive powers, line impendences, 
input system parameters and initialize variables

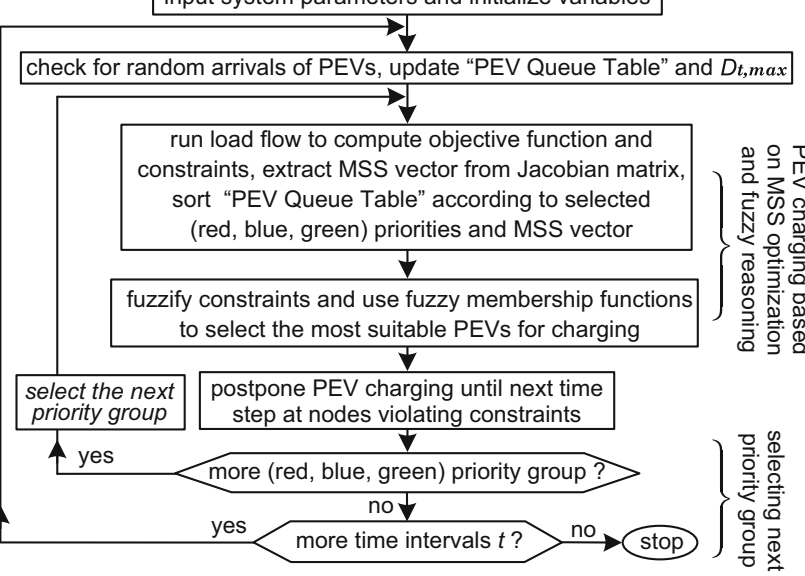

Fig. 3 Flow chart of OL-FCA for online PEV charging coordination

PEV types, ratings and descriptions) and initializing all variables. At each time interval $t=\Delta t, 2 \Delta t, \ldots .24$ hours, the OL-FC algorithm will take the following steps to determine the coordinated charging schedules of the randomly arriving PEVs:

- Step 1 (Run Load Flow and Compute Objective Function)- Sample the current state of the grid by the executing power flow calculations to compute the load levels, node voltages, line currents, system losses, etc. Compute objective function(Eq. 1) and MSS vectors (Eq. 3).

- Step 2 (Update PEV Queue Table)- Add the randomly arriving PEVs to the "PEV Queue Table". This table also contains PEVs from previous time steps that have not been charged due to a constraint violation. Sort the table from high to low priority based on the PEV time zones (red, blue, green) and sensitivities (Eq. 3). Use the PEV information in the queue table to update the value of $D_{\mathbf{t}, \mathbf{m a x}}$.

- Step 3 (Fuzzification of Constraints)- Fuzzify optimization constraints (Eq. 2) based on Eqs. 4-7 and Fig. 2.

- Step 4 (PEV Selection for Charging)- Select the most suitable PEVs for charging based on the solutions of the constrained optimization problem and the location of each vehicle in the "PEV Queue Table". Compute (starting with the PEV at the top of Queue Table) PEV fuzzy membership function (Eq. 7) and decide to either activate or delay (until next $t$ ) vehicle charging.

- Step 5 (Charging of Lower Priority PEVs)- Repeat Steps 1-4 for lower priority (blue and green) PEVs.

- Step 6 (Next Time Interval)- Perform Steps 1-5 for all time intervals within the 24 hours period.

The "PEV Queue Table" is an important part of the proposed PEV coordination algorithms that comprises of all randomly arriving PEVs at the current time step sorted from high to low priority. It also contains the PEVs from previous time steps that have not been charged due to distribution transformer overloading or voltage violations. The main program loop performs charging coordination continuously at every time interval starting with the vehicles listed on top of the PEV Queue Table. This table is updated at each time interval.

\section{Overnight MSS-Based Coordinated (ON-MSSC) Algorithm for PEV Charging [15]}

Reference [15] proposes an overnight MSS coordination algorithm (ON-MSSCA) for PEV charging that mostly looks after utility preference to perform valley filling during early morning hours while also reducing consumer charging cost and ensuring full charging of all PEV batteries for the next day trip. ON-MSSCA is based on the online PEV coordination approach of [9] with the following modifications:

- Vehicle charging during the peak load hours is not allowed. The information of the randomly arriving PEVs including their plug-in times and locations (nodes) are stored in the PEV Queue Table. The table is sorted according to the vehicle plug-in times and is updated at each time interval $\Delta t$.

- PEV charging starts and ends at designated off-peak load hours while the maximum demand level is set according to the total number of PEVs in the Queue Table:

$D_{t, \max }=\left(N_{P E V} \times E_{P E V}\right) /\left(T_{\text {start }}-T_{\text {end }}\right)$

where $T_{\text {start }}=24: 00 \mathrm{~h}$ and $T_{\text {end }}=06: 00 \mathrm{~h}$ are the designated starting and ending times for PEV charging while $N_{P E V}$ and $E_{P E V}$ indicate the total number of PEVs in the Queue Table at $t=T_{\text {start }}$ and energy required to charge each PEV.

\section{PEV Energy Requirement [15]}

The capacity of vehicle batteries is important for determining realistic PEV charging profiles. Typical PEV battery capacity varies from a few $\mathrm{kWh}$ to over $50 \mathrm{kWh}$. In this paper, a relatively small battery size of $10 \mathrm{kWh}$ is selected for each vehicle since it is expected that this size will be affordable and popular for EVs in the future. In order to optimize PEV battery life, deep cycle batteries with a depth of discharge (DOD) of $70 \%$ is assumed resulting in an available capacity of $7 \mathrm{kWh}$ that the charger must deliver. Since battery chargers comprise internal losses, the PEV energy 
requirement from the grid is actually greater than the actual battery capacity. In this paper, a typical battery charger efficiency of $88 \%$ requiring a total of $8 \mathrm{kWh}$ of energy from the grid to charge a single PEV is assumed.

\section{Consideration of Wind and Solar Distribution Generations in PEV Coordination Algorithms}

The OL-FC and ON-MSSC algorithms are modified to consider renewable wind and solar generations in their PEV charging strategies. This is done by dynamically adjusting the maximum demand level $D_{t, \max }$ of (2) based on the generation level of renewable DGs. Therefore, more PEVs are activated during the peak generation periods of wind DGs and rooftop PVs. The algorithm accommodates wind and solar generation by treating them as PQ nodes injecting only active power into the grid.

\section{Proposed Combined OL-F/DL-MSS PEV Coordination Strategy and Priority Groups}

To reduce the cost of purchasing or producing the energy required for vehicle charging, three charging time zones and three PEV coordination approaches are offered to consumers for quick, overnight and next day PEV charging (Fig. 1). A combined online fuzzy-based and delayed MSS-based (OL-F/DL-MSS) PEV coordination strategy is proposed that utilizes the online fuzzy algorithm of [14] and the overnight MSS algorithm of [15] with the addition of wind DGs and rooftop PVs. The OL-F/DL-MSS PEV coordination algorithm reduces energy generation cost by:

- Charging vehicles during off-peak load hours and maximum renewable energy generation periods.

- Dividing the consumers into high, low and medium priority groups and defining red, green and blue PEV charging time zones (Fig. 1).

\section{A. Red Charging Time Zone for Online Fuzzy Coordination of High Priority PEVs Considering Wind DGs}

The red charging time zone (1800h-2200h) is designed for high priority consumers requesting to charge their PEVs as soon as possible on return from work in order to have them ready for use later in the evening. These PEVs will be charged using the fast OL-FC algorithm and the owners will have to pay a higher tariff rate. The proposed OLF/DL-MSS will try to accommodate these PEVs as soon as they are randomly plugged-in while considering the cost minimization objective function and constraints. It will also try to charge as many vehicles as possible using inexpensive renewable energy provided by wind DGs.

\section{B. Green Charging Time Zone for Overnight MSS Coordination of Low Priority PEVs Considering Wind DGs}

The green charging time zone $(2400 \mathrm{~h}-0800 \mathrm{~h})$ is designed for the low priority consumers that prefer to charge their vehicles at partially off-peak periods and pay a cheap tariff rate. These consumers plug-in their vehicles as soon as coming home and require them to be charged for the next day trip by 0600h. These PEVs will be charged using the ON-MSSCA during the early morning hours with inexpensive electricity prices while performing valley filling. The proposed OL-F/DL-MSS will also try to charge as many vehicles as possible using inexpensive renewable energy provided by wind DGs.

\section{Blue Charging Time Zone for Daytime Fuzzy Coordination of PEVs Considering Solar (Rooftop) DGs}

The blue charging zone $(0800 \mathrm{~h}-1600 \mathrm{~h})$ is designed for vehicles that are plugged-in at homes, offices, industrial buildings and public PEV parking stations. The medium priority consumers usually will plug-in their vehicles in the morning and require them to be charged by $1600 \mathrm{~h}$. The proposed OL-F/DL-MSS algorithm will also try to charge as many vehicles as possible using inexpensive renewable energy provided by wind DGs and rooftop PVs.

\section{Simulation Results and Discussions}

For the simulation and analyses of this paper, the 449 node distributed SG topology of Fig. 4 is considered. It consists of the IEEE 31 node $23 \mathrm{kV}$ system $[9,14]$ with the addition of 3 wind DGs, 3 solar DGs and 22 low voltage 19 node 415 $\mathrm{V}$ residential feeders populated with PEVs. System parameters are provided in $[9,14]$. The penetration of PEVs is $63 \%$ corresponding to 264 vehicles. The numbers of PEVs in the red, green and blue charging time zones are 44, 88 and 132, respectively. The selected active output power characteristics of the wind and solar DGs that are used for the simulation of Fig. 4 are shown in Fig. 5.

The OL-F/DL-MSS PEV charging algorithm is used to coordinate PEVs during the 24-hour period. Four case studies are simulated and analyzed (Figs. 6 and 7):

- Case 1- Uncoordinated PEV charging without DGs.

- Case 2- Uncoordinated PEV charging with DGs. 


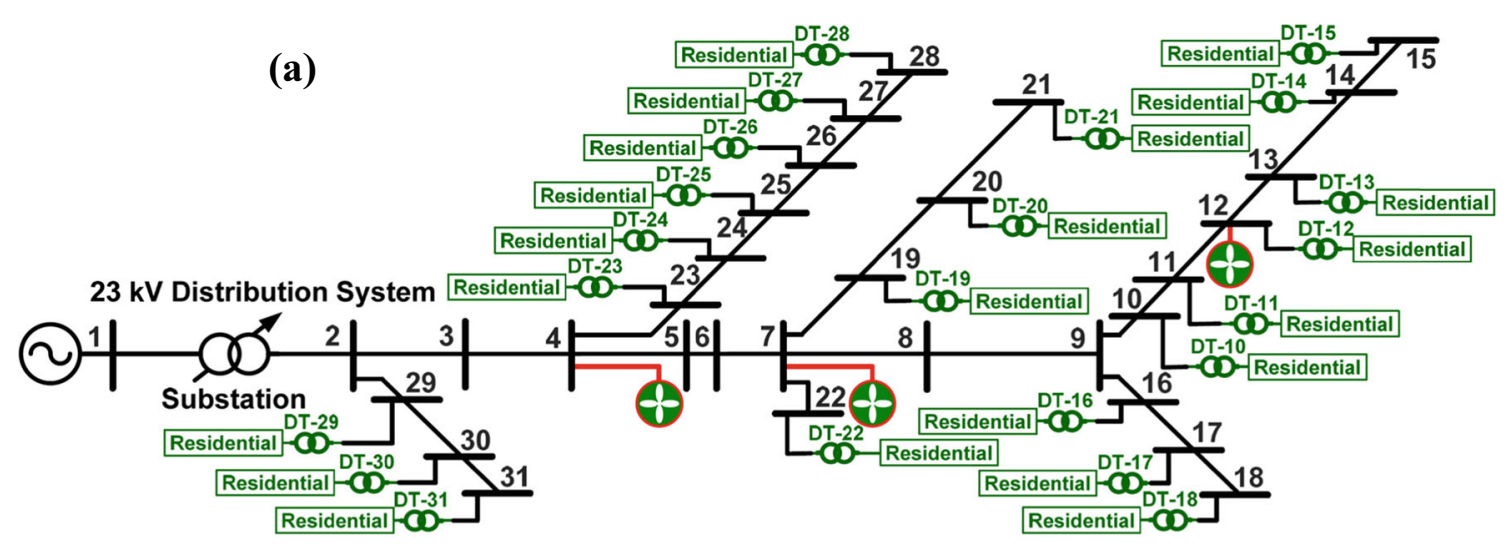

(b)

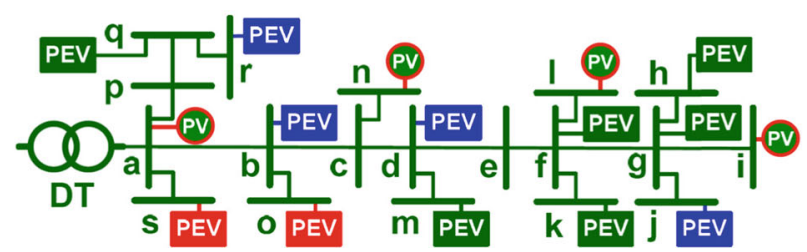

Fig. 4 The 449 node smart grid system consisting of the IEEE 31 node $23 \mathrm{kV}$ system with 3 wind DGs, 88 solar DGs (rooftop PVs) and 22 low voltage $415 \mathrm{~V}$ residential feeders populated with PEVs; a system diagram, $\mathbf{b}$ details of one residential feeder with $63 \% \mathrm{PEV}$ penetration (corresponding to the total of $264 \mathrm{PEVs}$ ) showing vehicles requesting red, green and blue charging time zones
- Case 3- Proposed combined OL-F/DL-MSS PEV charging scheme without DGs.

- Case 4- Proposed combined OL-F/DL-MSS PEV charging scheme with DGs.

Figures 6 and 7 and Table 1 present the impacts of uncoordinated and proposed coordinated (OL-F/DL-MSS) PEV charging on node voltage profiles, substation transformer loading, generation cost and total cost.

\section{Uncoordinated PEV Charging without and with Wind DGs and Rooftop PVs (Cases 1-2)}

To investigate the impacts of uncoordinated charging on the grid, charging scenarios with $16 \%, 32 \%, 47 \%$ and $63 \%$
PEV penetrations are simulated without (Case 1) and with (Case 2) wind DGs and rooftop PVs (Fig. 6). For these cases, PEVs are being randomly plugged in during early evening hours (1800h-2200h). Simulation results are summarized in Table 1 (rows 4-13) and plotted in Fig. 6 where:

- $\quad$ Fig. 6a and b present the daily load curve for $63 \% \mathrm{PEV}$ penetration without and with DGs, respectively.

- Fig. 6c and d present the substation transformer loading for $16 \%, 32 \%, 47 \%$ and $63 \% \mathrm{PEV}$ penetrations without and with DGs, respectively.

- $\quad$ Fig. 6e and f present the system losses for $16 \%, 32 \%$, $47 \%$ and $63 \% \mathrm{PEV}$ penetrations without and with DGs, respectively.
Fig. 5 The renewable output power characteristics used for the simulation of Fig. 4; a for wind DGs based on scaled down actual recordings from Walkway Wind Farm, WA, Australia on July 7, 2012 [14], b for solar DGs (rooftop PVs) based on scaled down actual recordings of daily summer and winter rooftop PV generations in Perth, WA, Australia [32] (a)

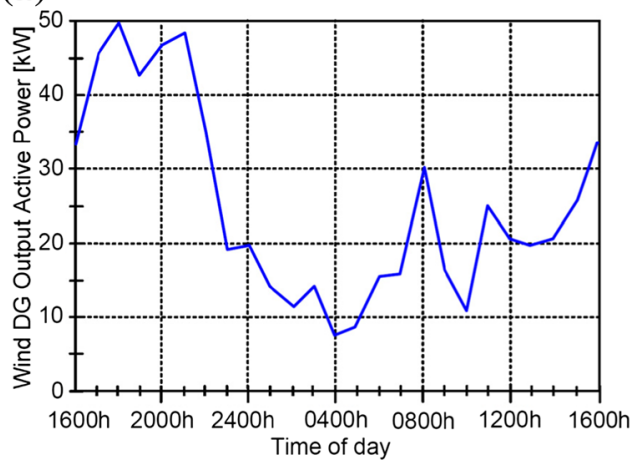

(b)

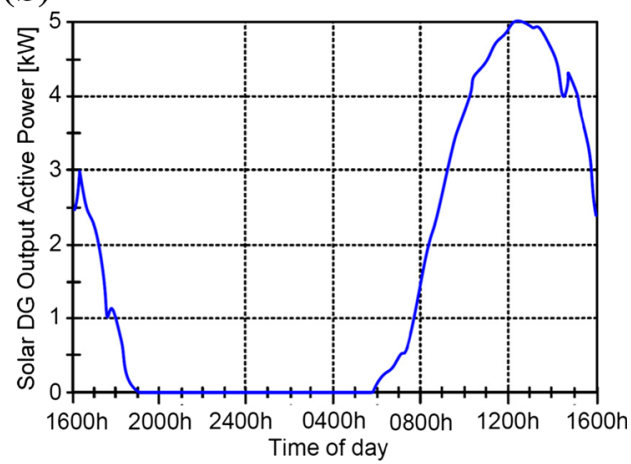


(a)

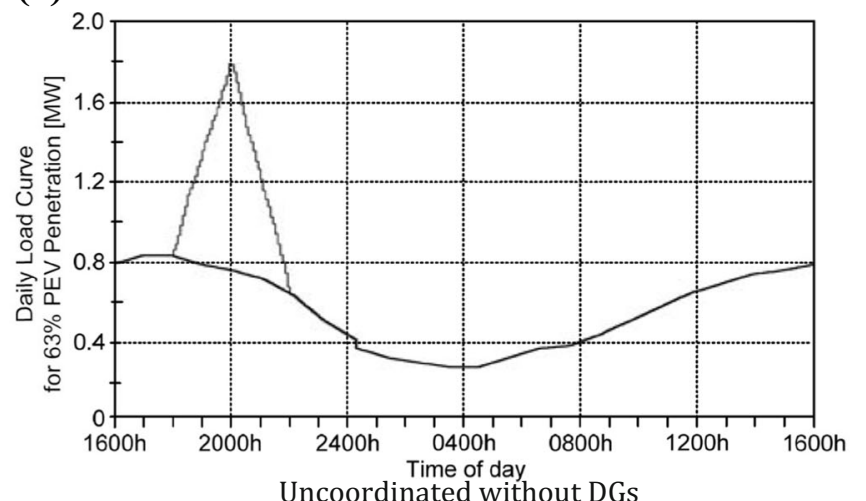

(c)

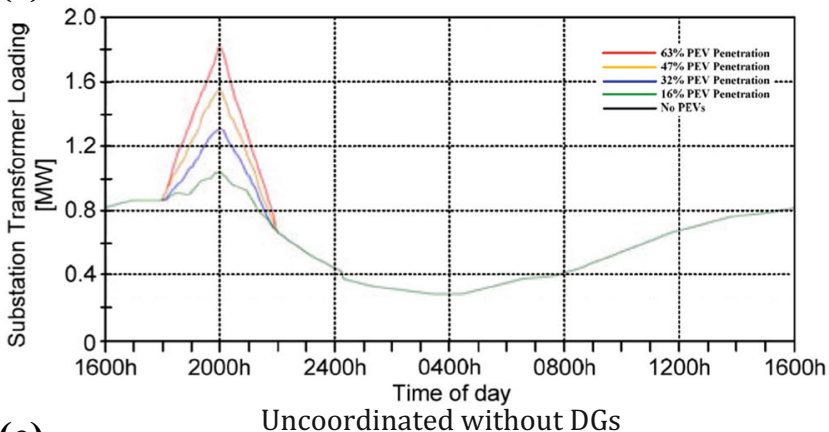

(e)

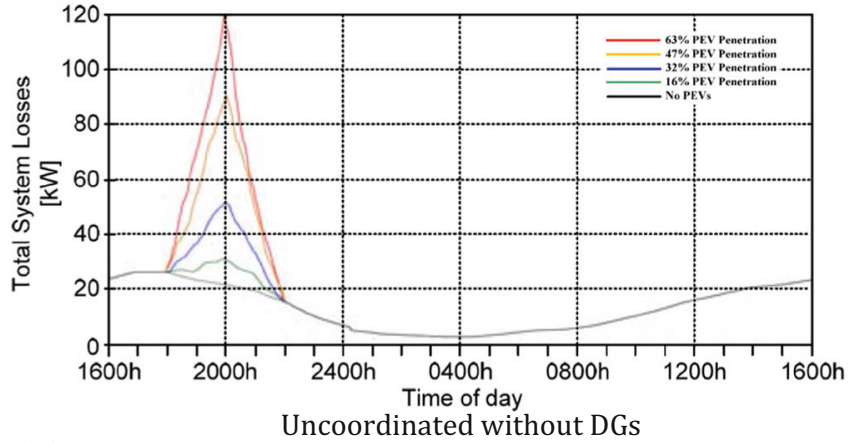

(g)

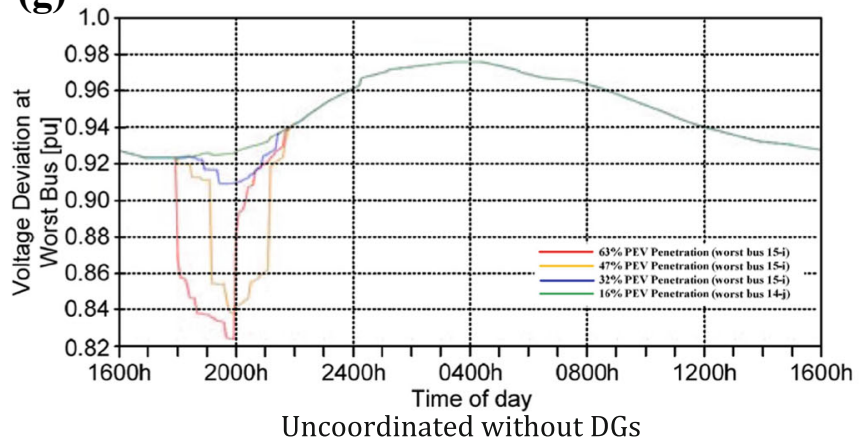

(b)

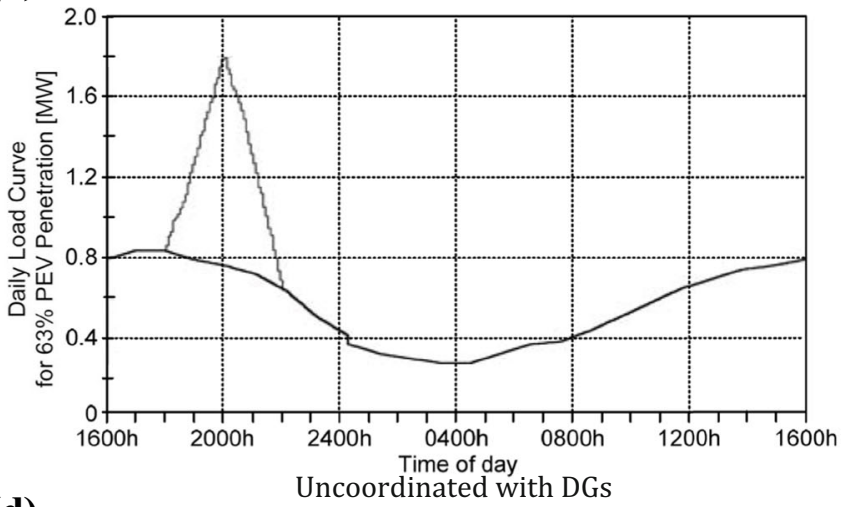

(d)

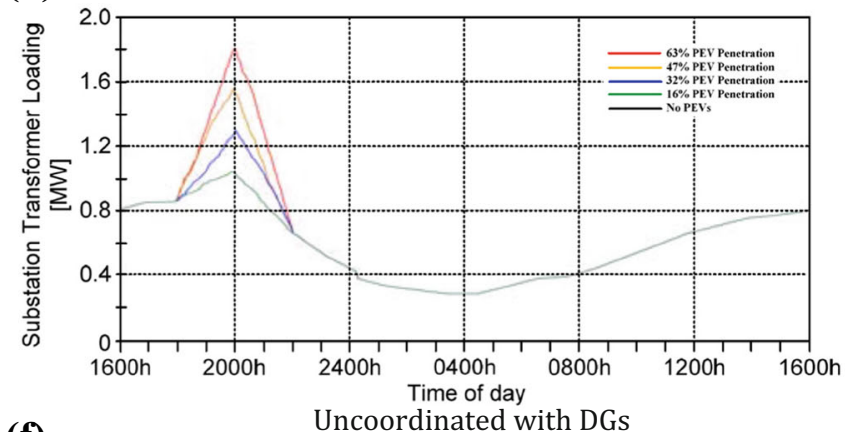

(f)

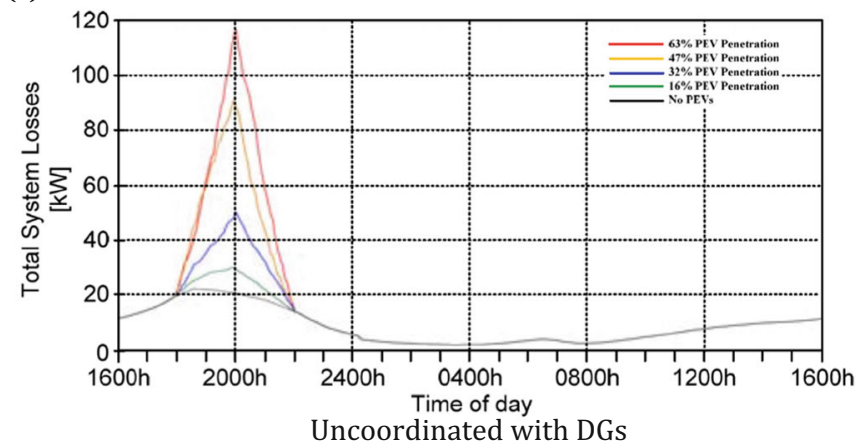

(h)

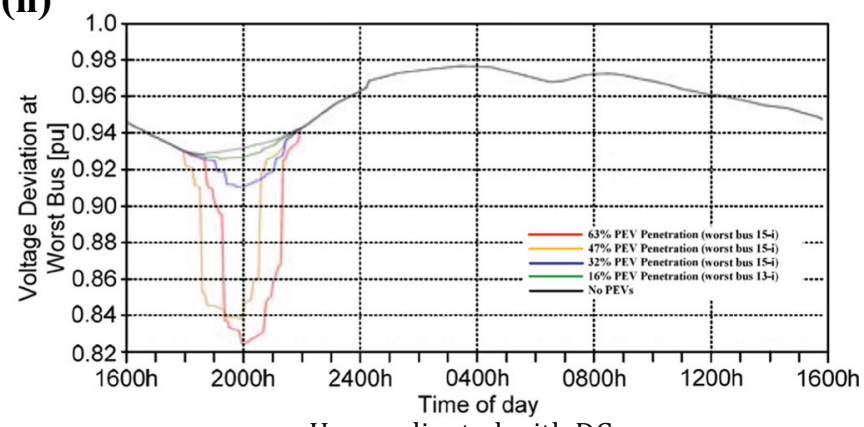

Uncoordinated with DGs

Fig. 6 Uncoordinated PEV charging (Cases 1-2); a-b Daily load curve for $63 \%$ PEV penetration without and with DGs, c-d Substation Transformer loading without and with DGs, (e)-(f) System losses without and with DGs, g-h Voltage profiles at worst bus without DGs and with DGs

- Fig. $6 \mathrm{~g}$ and $\mathrm{h}$ present the voltage profiles at worst bus for $16 \%, 32 \%, 47 \%$ and $63 \%$ PEV penetrations without DGs and with DGs, respectively.
As expected, there are significant increases in power demand, power generation, voltage deviations and power losses even at low PEV penetration levels. For example, 
(a)

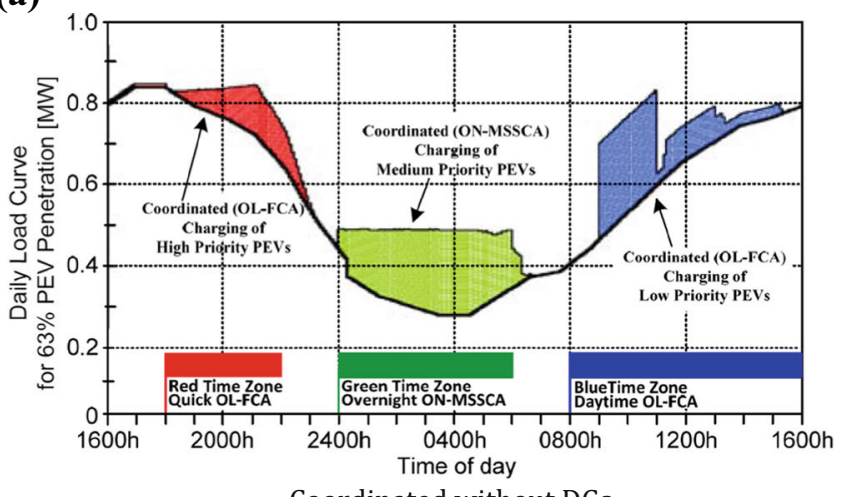

(c)

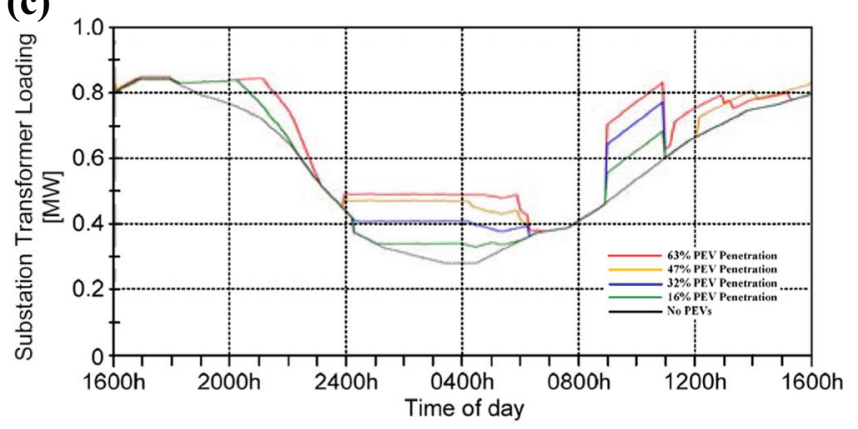

(e)

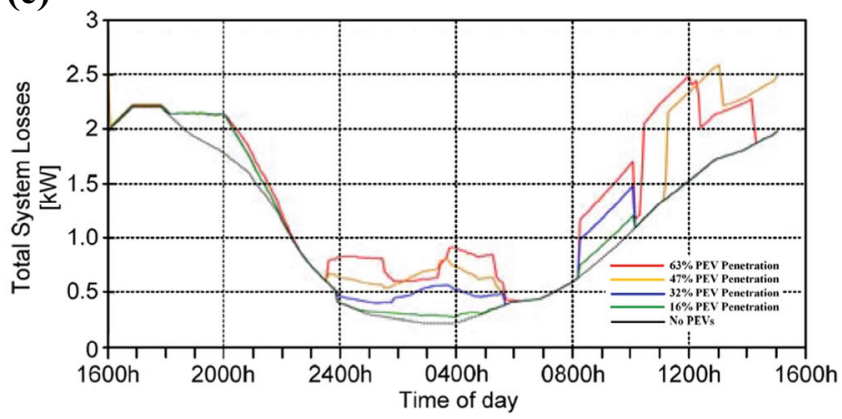

(g)

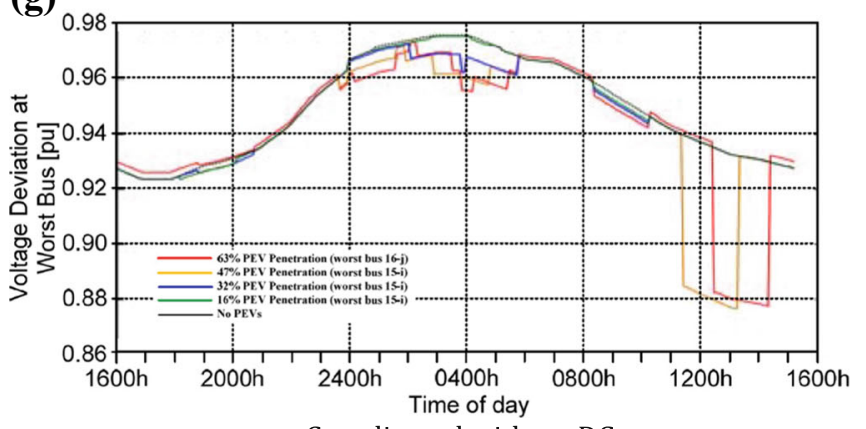

Coordinated without DGs

Fig. 7 Proposed combined OL-F/DL-MSS PEV charging strategy (Cases 3-4); a-b Daily load curve for $63 \%$ PEV penetration without and with DGs, c-d Substation Transformer loading without and with

total costs without DGs are increased by $30.8 \%$ and $59 \%$ for PEV penetration levels of $16 \%$ and $63 \%$, respectively (Table 1). The introduction of Wind DGs and rooftop PVs (b)

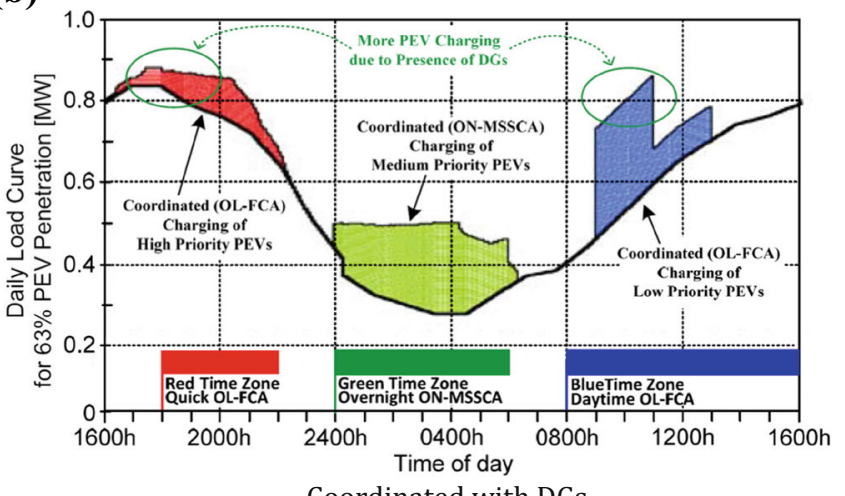

(d)

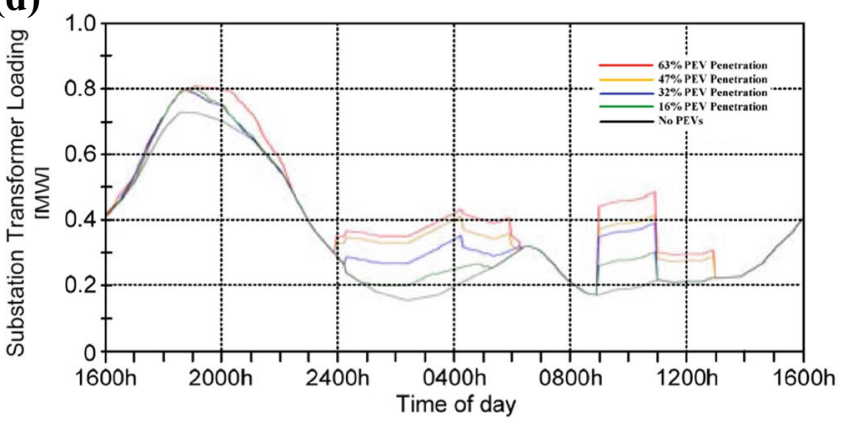

(f)

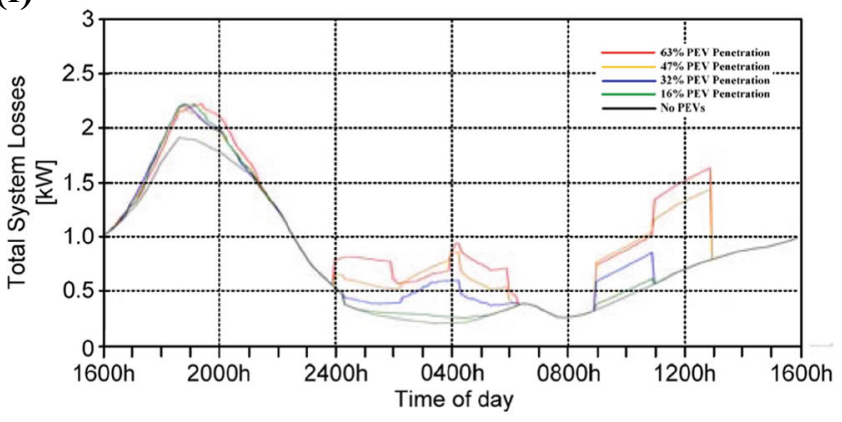

Coordinated with DGs

(h)

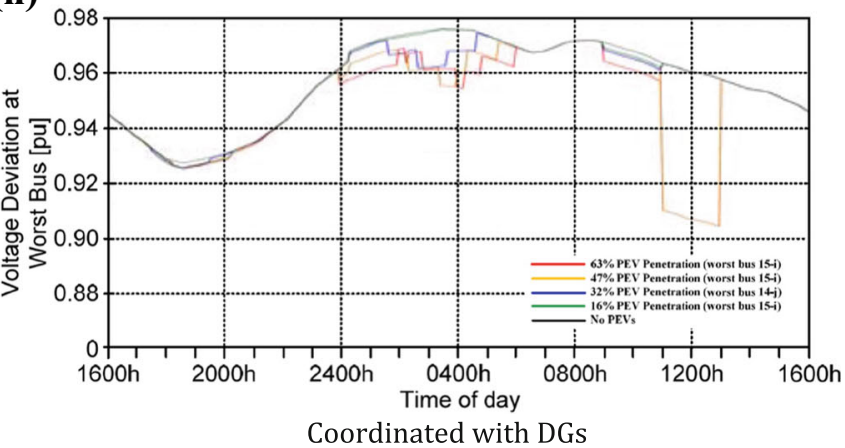

DGs, e-f System losses without and with DGs, $\mathbf{g}-\mathbf{h}$ Voltage profiles at worst bus without DGs and with DGs

has reduced the above-mentioned values to $-16.8 \%$ and $11.4 \%$. These reductions are mainly due to the presence of wind DGs during the early evening hours (Fig. 5a). 
Table 1 Impact of uncoordinated and proposed coordinated (combined OL-F/DL-MSS) PEV charging on smart grid system of Fig. 4

\begin{tabular}{llllll}
\hline PEV & $\Delta V$ & $I_{\mathbf{M A X}}$ & Generation & Total cost (Eq. 1) & Increase in \\
{$[\%]$} & {$[\%]$} & {$[\%]$} & cost $^{\mathrm{a}}$ & {$[\$ /$ day $]$} & $\begin{array}{l}\text { Total cost } \\
{[\%]^{\mathrm{b}}}\end{array}$
\end{tabular}

Nominal Operation without any PEVs or WDGs

$\begin{array}{llllll}0 & 7.646 & 0.147 & 770.3 & 786.2 & 0\end{array}$

Uncoordinated PEV Charging without DGs (Fig. 6a, c, e, g)

\begin{tabular}{|c|c|c|c|c|c|}
\hline 16 & 7.73 & 0.181 & 828 & 1,028 & 30.8 \\
\hline 32 & 9.08 & 0.219 & 871 & 1,093 & 39.0 \\
\hline 47 & 16.20 & 0.265 & 915 & 1,182 & 50.3 \\
\hline 63 & 17.60 & 0.307 & 958 & 1,250 & 59.0 \\
\hline \multicolumn{6}{|c|}{ Uncoordinated PEV Charging with DGs (Fig. 6b, d, f, h) } \\
\hline 16 & 7.42 & 0.177 & 508 & 654 & -16.8 \\
\hline 32 & 8.96 & 0.216 & 551 & 719 & -8.5 \\
\hline 47 & 16.20 & 0.263 & 595 & 808 & 2.77 \\
\hline 63 & 17.60 & 0.306 & 639 & 876 & 11.4 \\
\hline
\end{tabular}

Proposed Combined OL-F/DL-MSS PEV Charging without DGs (Fig. 7a, c, e, g)

$\begin{array}{llllll}16 & 7.64 & 0.159 & 797 & 814 & 3.5\end{array}$

$\begin{array}{llllll}32 & 7.65 & 0.158 & 828 & 835 & 6.2\end{array}$

$\begin{array}{llllll}47 & 12.35 & 0.160 & 841 & 860 & 9.4\end{array}$

$\begin{array}{llllll}63 & 12.24 & 0.159 & 869 & 889 & 13.1\end{array}$

Proposed Combined OL-F/DL-MSS PEV Charging with DGs (Fig. 7b, d, f, h)

$\begin{array}{llllll}16 & 7.40 & 0.152 & 482 & 494 & -37.2 \\ 32 & 7.37 & 0.152 & 502 & 515 & -34.5 \\ 47 & 9.51 & 0.152 & 525 & 539 & -31.4 \\ 63 & 9.51 & 0.164 & 553 & 568 & -27.8\end{array}$

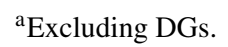

${ }^{b}$ Percentage of nominal cost with no PEVs, exculding DG costs.
It can be observed that the total system loss waveforms are very similar without (Fig. 6e) and with (Fig. 6f) the renewable DGs. This is due to the fact that the peak generation periods of renewable DGs (around $1700 \mathrm{~h}$ and $1200 \mathrm{~h}$ for wind and solar DGs, respectively; as shown in Fig. 5a-b) don't fully overlap with the peak PEV charging load (around 2000h as shown in Fig. 6a).

\section{Coordinated OL-F/DL-MSS PEV Charging Scheme without/with Wind DGs and Rooftop PVs (Cases 3-4)}

The proposed OL-FC/ON-MSSC is implemented and results are presented in Table 1 (rows 14-23) and Fig. 7. There is significant improvement in system operation and performance compared with the uncoordinated Cases 12. For example, there is a considerable improvement in the percentage increase of total cost (Table I, last column) with $63 \%$ PEV penetration from $59 \%$ for uncoordinated charging without DGs to $13.1 \%$ for coordinated charging without DGs and from $11.4 \%$ for uncoordinated charging with DGs to $-27.8 \%$ for coordinated charging with DGs while keeping node voltage profiles and maximum demand level within the permissible limits as. It is worth mentioning that, unlike the MSS-based overnight coordination (ON-MSSC), the fuzzy-based daytime coordination algorithm (OL-FCA) will allow small deviations/violations of (voltage and/or maximum demand) constraints (see Fig. $6 \mathrm{~g}$ ) according to the corresponding member functions of Fig. 2 to limit losses and reduce cost of generating energy.

Unlike the previous case, the total system loss waveforms are significantly different without (Fig. 7e) and with (Fig. 7f) the renewable DGs. This is a key advantage of the presented PEV coordination approach which is mainly due to its ability to delay the charging activities to the after peak hours and try to directly charge the PEV batteries from the renewable energy resources.

It is worth mentioning that the fuzzy logic PEV coordination method presented in this paper can be used for radial and non-radial distribution networks. However, there are other more efficient approaches for solving the convex PEV coordination problem associated with radial networks $[33,34]$. This paper does not consider the stochastic behavior of the wind and solar (rooftop PV) generations [35, 36]; 
however, the stochastic nature of EVs are included by storing the random plug-in times of each vehicle in the "PEV Queue Table" and updating them at each time interval.

\section{Conclusion}

This paper presents a combined online fuzzy-based and delayed MSS-based (OL-F/DL-MSS) algorithm that considers distributed wind and PV generations to reduce grid costs associated with the energy generation and losses while limiting node voltage variations and distribution transformer loading below their designated permissible levels. The proposed OL-F/DL-MSS algorithm is implemented based on:

- Classifying the PEV owners to three groups of high, medium and low priorities consumer willing to pay expensive, inexpensive and cheap energy prices for quick, overnight and next-day battery vehicle charging, receptively.

- Performs residential peak-load shaving while also reducing distribution transformer loading by encouraging and shifting PEV loads to off-peak hours (overnight and next-day).

- Reducing the possibility of reverse powering and overvoltage conditions by charging PEV batteries from the available distributed wind DGs and rooftop PVs.

Simulation studies and analyses are carried out on a 449 node SG consisting of 3 winds DGs and 22 low voltage 19node residential feeders with 88 rooftop PVs and 264 PEVs. The main conclusions are:

- Compared to uncoordinated PEV charging, OL-F/DLMSS offer significant improvements in terms of system losses, voltage profiles and cost reduction.

- OL-F/DL-MSS takes advantage of wind DGs and rooftop PVs by utilizing their generated power particularly during peak generation periods to serve more vehicles, reduce the total cost and decreases the loading and stress on the distribution and substation transformers.

- OL-F/DL-MSS offers selections of three charging time zones based on consumer's priority.

- With OL-F/DL-MSS, the consumer also has the advantage to select his/her own preferred PEV coordination schemes including fast expensive charging during early evening hours, overnight cheap charging and daylight charging with moderate rates.

- OL-F/DL-MSS will also reduce the burden on distribution and substation transformers that will minimize the cost of premature asset failure and associated outages.

\section{References}

1. Fang X, Misra S, Xue G, Yang D (2012) Smart grid- the new and improved power grid: A survey. IEEE Commun Surv Tutorials 14(4):944-980. Fourth Quarter

2. Yilmaz M, Krein PT (2013) Review of the impacts of vehiclesto-grid technologies on distribution systems and utility interfaces. IEEE Trans Power Electron 28(12):5673-5689

3. Fernández LP, Román TGS, Cossent R, Domingo CM, Frías P (2011) Assessment of impact of plug-in electric vehicles on distribution networks. IEEE Trans Smart Grid 26(1):206-213

4. Sortomme E, Hindi MM, MacPherson SDJ, Venkata SS (2011) Coordinated charging of plug-in hybrid electric vehicles to minimize distribution system losses. IEEE Trans Smart Grid 2(1):198-205

5. Zhongjing M, Callaway DS, Hiskens IA (2013) Decentralized charging control of large populations of plug-in electric vehicles. IEEE Trans Control Syst Technol 21(1):67-78

6. Vandael S, Claessens B, Hommelberg M, Holvoet T, Deconinck G (2013) A scalable three-step approach for demand side management of plug-in hybrid vehicles. IEEE Trans Smart Grid 4(2):720 728

7. Jin C, Tang J, Ghosh P (2013) Optimizing electric vehicle charging: A customer's perspective. IEEE Trans Veh Technol 62(7):2919-2927

8. Foster JM, Caramanis MC (2013) Optimal power market participation of plug-in vehicles pooled by distribution feeder. IEEE Trans Power Syst 28(3):2065-2076

9. Deilami S, Masoum AS, Moses PS, Masoum MAS (2011) Realtime coordination of plug-in electric vehicle charging in smart grids to minimize power losses and improve voltage profile. IEEE Trans Smart Grid 2(3):456-467

10. Wu D, Aliprantis DC, Ying L (2012) Load scheduling and dispatch for aggregators of plug-in electric vehicles. IEEE Trans Smart Grid 3(1):368-376

11. Geng B, Mills JK, Sun D (2013) Two-stage charging strategy for plug-in electric vehicles at the residential transformer level. IEEE Trans Smart Grid 4(3): 1442-1452

12. Gan L, Topcu U, Low S (2013) Optimal decentralized protocol for electric vehicle charging. IEEE Trans Power Syst 28(2):940-951

13. Richardson P, Flynn D, Keane A (2012) Optimal charging of electric vehicles in low-voltage distribution systems. IEEE Trans Power Syst 27(1):268-279

14. Amir S, Masoum S, Deilami A, Abu-Siada MAS (2015) Masoum, Fuzzy logic approach for online coordination of charging plug-in electric vehicles in smart grids. IEEE Trans Sustainable Energy 6(3):112-1121

15. Masoum AS, Deilami S, Masoum MAS, Abu-Siada A, Islam SM (2014) Overnight coordinated charging of plug-in electric vehicles based on maximum sensitivities selections, Applied Engineering Sciences. In: Wei Deng (ed), vol 65-70. CRC Press, London UK

16. Bashash S, Fathy HK (2012) Transport-based load modeling and sliding mode control of plug-in electric vehicles for robust renewable power tracking. IEEE Trans Smart Grid 3(1):526-534

17. Falahi JM, Hung-Ming Chou C, Ehsani M, Le X, Butler-Purry KL (2013) Potential power quality benefits of electric vehicles. IEEE Trans Sustainable Energy 2(1):1016-1023

18. Pillai JR, Bak-Jensen B (2011) Integration of vehicle-to-grid in the Western Danish power system. IEEE Trans Sustainable Energy 2(1):12-19

19. Hu W, Su C, Chen Z, Bak-Jensen B (2013) Optimal operation of plug-in electric vehicles in power systems with high wind power penetrations. IEEE Trans Sustainable Energy 2(1):577-585

20. Vaya MG, Andersson G (2016) Self-scheduling of plug-in electric vehicle aggregator to provide balancing services for wind power. IEEE Trans Sustainable Energy 7(2):886-889 
21. Kou P, Liang D, Gao L, Gao F (2016) Stochastic coordination of plug-in electric vehicles and wind turbines in microgrid: A model predictive control approach. IEEE Trans Smart Grid 7(3):1537-1551

22. Sheppard CJR, Harris A, Gopal AR (2016) Cost-Effective Siting of Electric Vehicle Charging Infrastructure With Agent-Based Modeling. IEEE Trans Transport Elect 2(2):174-189

23. Sun B, Dragičević T, Freijedo FD, Vasquez JC, Guerrero JM (2016) A Control Algorithm for Electric Vehicle Fast Charging Stations Equipped With Flywheel Energy Storage Systems. IEEE Trans Power Electron 31(9):6674-6685

24. Masoum AS, Deilami S, Moses PS, Masoum MAS, Abu-Siada A (2011) Smart Load Management of Plug-in Electric Vehicles in Distribution and Residential Networks with Charging Stations for Peak Shaving and Loss Minimisation Considering Voltage Regulation. IET Gener Transm Distrib 5(8):877-888

25. Akhavan-Rezai E, Shaaban MF, El-Saadany EF, Karray F (2016) Online Intelligent Demand Management of Plug-In Electric Vehicles in Future Smart Parking Lots. IEEE Syst J 10(2):483-494

26. Yao W, Chung CY, Wen F, Qin M, Xue Y (2016) ScenarioBased Comprehensive Expansion Planning for Distribution Systems Considering Integration of Plug-in Electric Vehicles. IEEE Trans Power Syst 31(1):317-328

27. Xu Z, Su W, Hu Z, Song Y, Zhang H (2016) A Hierarchical Framework for Coordinated Charging of Plug-In Electric Vehicles in China. IEEE Trans Smart Grid 7(1):428-438
28. Tabari M, Yazdani A (2016) An Energy Management Strategy for a DC Distribution System for Power System Integration of Plug-In Electric Vehicles. IEEE Trans Smart Grid 7(2): 659-668

29. Momber I, Wogrin S, Román TGS (2016) Retail Pricing: A Bilevel Program for PEV Aggregator Decisions Using Indirect Load Control. IEEE Trans Power Syst 31(1):464-473

30. Baccino F, Grillo S, Massucco S, Silvestro F (2016) Behavioral Characterization of Electric Vehicle Charging Loads in a Distribution Power Grid Through Modeling of Battery Chargers. IEEE Trans Smart Grid 6(2):759-766

31. Haidar AMA, Muttaqi KM (2016) A Two-Stage Margin-Based Algorithm for Optimal Plug-in Electric Vehicles Scheduling. IEEE Trans Ind Appl 52(1):483-492

32. http://www.perthsolarcity.com.au/

33. Gan L, Topcu U, Low S (2013) Optimal decentralized protocol for electric vehicle charging. IEEE Trans Power Syst 28(2):940-951

34. Zhang B, Tse D (2013) Geometry of injection regions of power networks. IEEE Trans Power Syst 28(2):788-797

35. Wang Y, Wang B, Chu CC, Pota HR, Gadh R (2016) Energy management for a commercial building microgrid with stationary and mobile battery storage. Energ Buildings 116:141-150

36. Bai X, Qiao W (2015) Robust optimization for bidirectional dispatch coordination of large-scale V2G. IEEE Trans Smart Grid 6(4):1944-1954 